
R. WEIGAND ${ }^{1, \infty}$
H. CRESPO ${ }^{2}$
R. SASTRE ${ }^{3}$

\title{
Study of the broad-band saturable absorption of indocyanine green J-aggregates in polymeric films using 10-fs laser pulses
}

\author{
${ }^{1}$ Departamento de Óptica, Facultad de Ciencias Físicas, Universidad Complutense de Madrid, \\ Ciudad Universitaria s/n, 28040 Madrid, Spain \\ ${ }^{2}$ CLOQ/Departamento de Física, Faculdade de Ciências, Universidade do Porto, \\ Rua do Campo Alegre, 687, 4169-007 Porto, Portugal \\ ${ }^{3}$ Instituto de Ciencia y Tecnología de Polímeros, CSIC (Spain), C/Juan de la Cierva 3, 28006 Madrid, Spain
}

\begin{abstract}
Received: 17 June 2005/Revised version: 11 November 2005 Published online: 17 December 2005 • ( ) Springer-Verlag 2005

ABSTRACT Thin films of indocyanine green J-aggregates in polymers have been prepared and their saturable absorption properties studied in a high intensity regime using a unique ultrashort pulse Ti:sapphire laser (10 fs) with a very broad bandwidth (over $200 \mathrm{~nm}$ at $-20 \mathrm{~dB}$ ). The samples showed a surprisingly high stability to irradiation, strong absorption bleaching, and wavelength dependent differential absorption spectra.
\end{abstract}

PACS $42.70 \mathrm{Jk}$; 42.65.-k; 78.66.Qu

1

\section{Introduction}

The J-aggregates are systems which have had a growing interest since its discovery by Scheibe and Jelly in 1934, especially in the field of nonlinear optics. Their properties are given by the excitonic nature of their identifiable J-band. Among the nonlinear properties of the J-band is its saturable absorption which has been extensively studied in many J-aggregates in solution, as well as in thin films. This property has been applied to optical switches $[1,2]$ and for passive mode-locking in lasers [3]. The J-band of indocyanine green (ICG) aggregates in water solution lies in the infrared zone $(890 \mathrm{~nm}$ ) [4] and its saturable absorption properties have already been studied [5]. Part of the interest in this compound is that the central absorption wavelength of its J-band can be reached for certain operation conditions of a Ti:sapphire laser, and thus the nonlinear properties can be thoroughly studied. Previous studies were made using with ultrafast lasers with typically 80 fs pulse durations, corresponding approximately to a spectral range of only $4 \mathrm{~nm}$ for transform limited pulses. The unusual characteristics of the Ti:sapphire laser used in this work, namely a much broader spectra and few-cycle pulse durations [6] are adequate for the study of the nonlinear optical properties of the J-aggregates in a broad spectral zone around their absorption band. Since the solid state media is much more convenient than liquid solutions and, to our knowledge, no studies in the solid form of ICG have been performed, this work is focused on the broad-band saturable

Fax: +34-91-3944683, E-mail: weigand@ fis.ucm.es absorption characteristics of ICG J-aggregates embedded in polymeric thin films.

\section{$2 \quad$ Experimental \\ 2.1 Sample preparation}

$\mathrm{J}$-aggregates of ICG in water were prepared departing from $1.5 \times 10^{-3} \mathrm{M}$ solutions heated at $70^{\circ} \mathrm{C}$ in an electric oven during $72 \mathrm{~h} \mathrm{[4].} \mathrm{In} \mathrm{order} \mathrm{to} \mathrm{carry} \mathrm{out} \mathrm{the} \mathrm{study} \mathrm{in} \mathrm{thin}$ films, different polyvinyl-alcohol polymers (PVA) were selected as matrices. The PVA polymer was chosen because both polarity and solubility were appropriate to mimic water as the solvent. The preliminary results obtained in the preparation of thin films with ICG showed that the most suitable among them was a PVA with an average molecular weight number of $M n=17200$, corresponding to a polymerization degree of 350 and a hydrolysis degree of $87-89 \mathrm{~mol} \%$. The PVA is obtained by hydrolysis of polyvinyl acetate. Thus the hydrolysis degree in PVA represents the number of hydroxil groups per one hundred repetition units in the polymer, that is, the percentage of repetition monomer units (vinyl alcohol) in the final polymer. The water solution was mixed in $a: b$ proportions with a solution of $0.4 \mathrm{~g} / 20 \mathrm{~cm}^{3}$ of this PVA ( $a=$ volume of J-aggregate solution, $b=$ volume of PVA), and some drops were deposited in a Petri cell. The film resulted from evaporation of the solvents at room temperature and air atmosphere and thus the resulting J-aggregates are not expected to be oriented. Two different series of samples were prepared with the aim of reaching the highest possible absorbance in the J-band. In the first series, concentration was changed and hence we shall call it series $\mathrm{C}$. It was performed with volume proportions $a: b=0.75: 9$ for sample $\mathrm{C} 1$ and $1: 9$ for sample $\mathrm{C} 2$. The second series was done with different thicknesses and hence we shall call it series T. We used proportions $a: b=1: 9$ and made samples having 1,2,3 layers (samples T1, T2, T3), where each consecutive layer was fabricated after the previous one had dried up. The thickness of the film was not controlled during fabrication and was later measured with a non contact confocal imaging profiler using a $50 \times$ microscope objective. Series $C$ had thicknesses ranging from $1 \mu \mathrm{m}$ to $10 \mu \mathrm{m}$, while series T had from $10 \mu \mathrm{m}$ to $25 \mu \mathrm{m}$. Figure 1 shows a picture of the films in series $\mathrm{C}$ for the two different concentrations, and in series $\mathrm{T}$ for the three different 

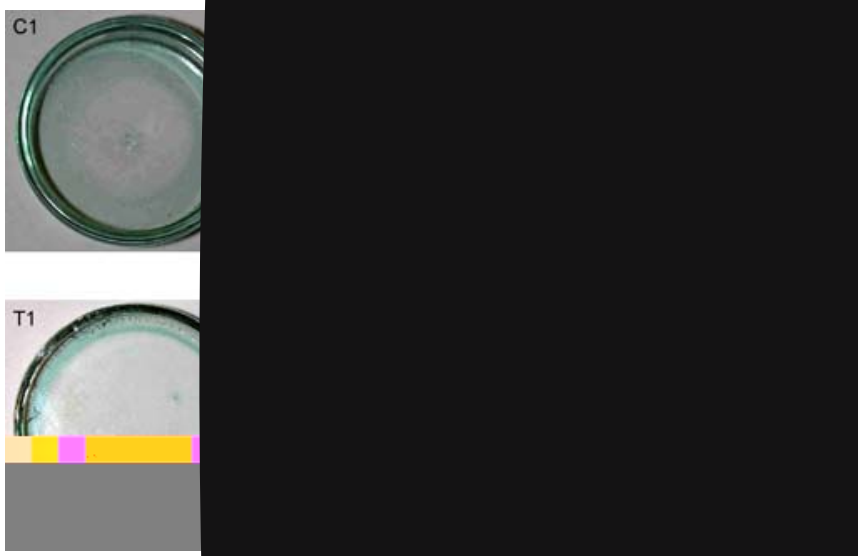

FIGURE 1 Photographs of the samples used

thicknesses; spatial inhomogeneities become more and more evident as the aggregate concentration increases.

In order to check on the integrity of the aggregate throughout the preparation procedure with the polymers, linear absorption spectra of the final samples were taken using a double-beam spectrophotometer and measuring the sample with air as reference. The transmittance of the samples is shown in Fig. 2. We clearly see that the J-band at $890 \mathrm{~nm}$ indicative of the J-aggregate of ICG in solution (Fig. 2a) is shifted to $875 \mathrm{~nm}$ in the thin films (Fig. $2 \mathrm{~b}$ and c), probably due to a strong interaction with the polymer. Also, oligomeric forms seem to appear for the more concentrated samples (Fig. 2c, T3). The absence of cells in the reference beam of the spectrometer, scattering and low optical quality of the samples can account for the absence of $100 \%$ transmission, far from the absorption band.

\subsection{Stability measurements}

Since for saturable absorption measurements high irradiances are required, stability measurements of the samples were performed in conditions corresponding to the highest optical intensity that will be used for the saturable absorption measurements. The experimental set-up used is depicted in Fig. 3. A laser beam from a home-built Ti:sapphire laser oscillator [6] $\left(85 \mathrm{MHz}\right.$ repetition rate, $\lambda=800 \mathrm{~nm}, \Delta \lambda_{\mathrm{L}}=$ $200 \mathrm{~nm}$ bandwidth at $-20 \mathrm{~dB}, 85 \mathrm{~mW}$ output power and $10 \mathrm{fs}$ pulse duration) was employed. The extremely broad spectrum of the laser is represented in Fig. 4. Ultrashort laser pulses are usually very sensitive to any ambient changes, such as temperature and air flow, since the corresponding changes in optical path will influence the dispersion compensation in the laser cavity thus, affecting the spectral output of the laser, and the two plots in Fig. 4 show the differences that ap-

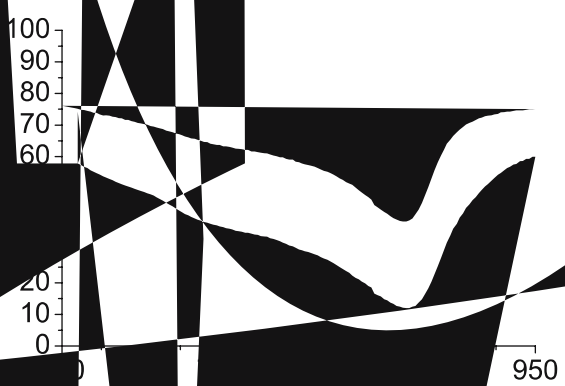

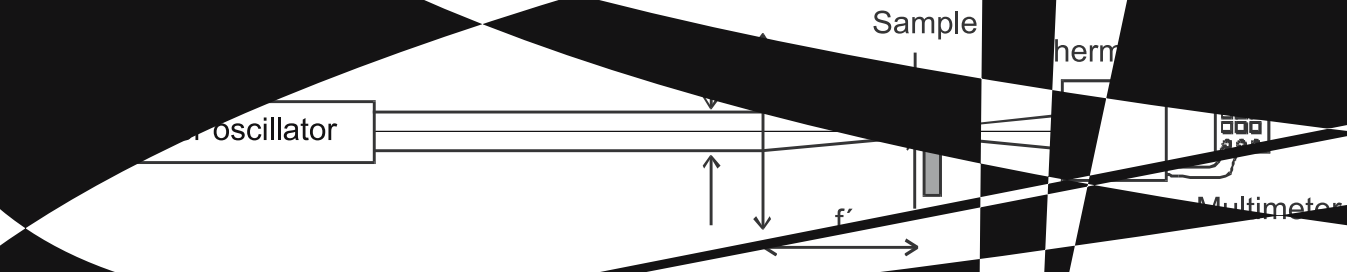

FIGURE 3 Experimental setup for stability measurements 
$\begin{array}{lllllll}650 & 700 & 750 & 800 & 850 & 900 & 950\end{array}$ 


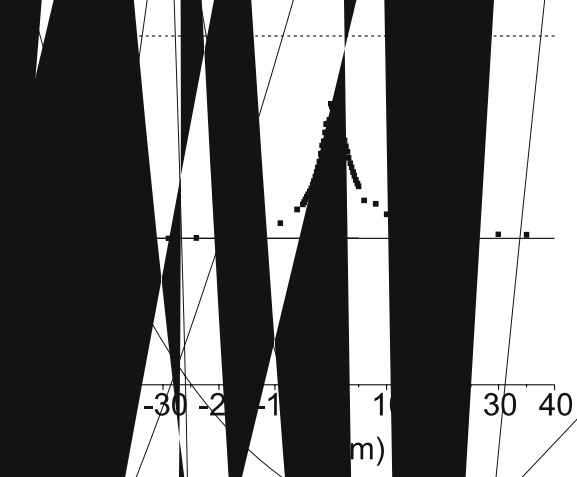

$-40-30-20-10 \quad 0 \quad 10$ 
son along the whole spectral range. For all samples it can be clearly seen how the nonlinear transmitted power is higher in the zone where the J-band lies (800-900 nm).

An even more informative picture is given by Fig. 8 where we plot the ratio of the nonlinear transmitted intensity vs. linear transmitted intensity (NLTP/LTP). This ratio is clearly wavelength dependent, except for the sample with the polymer alone. Wavelength dependent nonlinear properties in Jaggregates under high peak intensities $\left(100 \mathrm{MW} / \mathrm{cm}^{2}\right)$ have been previously observed by other authors $[8,9]$. Specifically, a blue-shift of a few nanometers was observed in the differential absorption spectrum (DAS is the change of optical density $\triangle \mathrm{OD}$, and hence the relationship with our measurements is $\mathrm{DAS}=\Delta \mathrm{OD}=\mathrm{OD}_{\mathrm{NL}}-\mathrm{OD}_{\mathrm{L}}=-\log _{10}($ 
ACKNOWLEDGEMENTS Part of this work has been financed by Project BFM2000-0796 from Ministerio de Ciencia y Tecnología (Spain). This work was also partially supported by FCT (Portugal) and FEDER through Grants No. POCTI/FAT/41900 and No. POCTI/FAT/48709.

R. Weigand thanks Ministerio de Educación y Ciencia for the financial support ("Programa Movilidad del Profesorado", Reference:PR2004-0017) and Prof. Dr. L. Bernardo for accepting her visit to CLOQ.

\section{REFERENCES}

1 L.S. Pu, Opt. Mater. 21, 489 (2002)

2 H. Kawashima, M. Furuki, S. Tatsuura, M. Tian, Y. Sato, L.S. Pu, T. Tani, Appl. Phys. Lett. 77, 1283 (2000)

3 A.S. Kuchýanov, R.V. Markov, A.I. Plekhanov, A.E. Simanchuk, V.I. Avdeeva, B.I. Shapiro, Y.I. Slominskii, A.I. Tolmachev, Opt. Commun. 231, 343 (2004)

4 F. Rotermund, R. Weigand, A. Penzkofer, Chem Phys. 220, 385 (1997)
5 M. Wittmann, F. Rotermund, R. Weigand, A. Penzkofer, Appl. Phys. B 66, 453 (1998)

6 H. Crespo, M.A. Cataluna, A. Guerreiro, J.T. Mendonça, High-order self-dispersion compensation in a Ti:sapphire laser oscillator, in $\mathrm{Ul}$ trafast Phenomena XIII, Springer Series in Chem. Phys., Vol. 71, D.R. Miller, M.M. Murnane, N.F. Scherer, A.M. Weiner (Eds.), (Springer 2002), pp 161-163

7 R. Weigand, F. Rotermund, A. Penzkofer, J. Phys. Chem. 101, 7729 (1997)

8 R. Gadonas, K.H. Feller, A. Pugzlys, Opt. Commun. 112, 157 (1994)

9 R. Gadonas, K.H. Feller, A. Pugzlys, G. Jonusauskas, J. Oberlé, C. Ruillière, J. Phys. Chem. 106, 8374 (1997)

10 F. Sasaki, S. Kobayashi, J. Lumin. 72-74, 538 (1997)

11 Y. Hamanaka, H. Kurasawa, A. Nakamura, Y. Uchiyama, K. Marumoto, S. Kuroda, Chem. Phys. Lett. 363, 233 (2002)

12 K.H. Feller, E. Gaižauskas, J. Lumin. 83-84, 265 (1999)

13 E. Gaižauskas, K.H. Feller, J. Molec. Struct. 598, 45 (2001)

14 E. Gaižauskas, K.H. Feller. Opt. Commun. 216, 217 (2003) 